\title{
El proceso de autoevaluación y autoregulación de la Sede del Atlántico de la Universidad de Costa Rica: Una mirada a la opinión de sus participantes tres años después
}

\author{
Susan Francis Salazar \\ Viria Ureña Salazar
}

\begin{abstract}
Resumen: El autoestudio y la autovaloración como estrategias evaluativas para los diferentes sistemas de educación superior han creado la necesidad de abrir espacios para la revisión y análisis de experiencias vividas; como es la que desarrolló la Sede del Atlántico, en 1996, como parte del Programa Cre-Columbus y patrocinado por la Vicerrectoría de Docencia.

Este trabajo intenta hacer una revisión y análisis de las opiniones de los participantes del proceso de Autoevaluación y Autorregulación vivido en la Sede durante los años 1996-1997 y la implementación de los proyectos que se originaron como parte del Plan de Acción, generando así una serie de consideraciones importantes dentro de los procesos de evaluación en la educación superior.
\end{abstract}

\section{Introducción}

La puesta en práctica de nuevas estrategias evaluativas en la educación superior ha privilegiado la autoevaluación y la autorregulación como prácticas modernas, eficientes e innovadoras en la evaluación universitaria. (Castillo, 2000). El proceso de autoevaluación se define, según Martín (1994, p. 8) como "un proceso de evaluación donde los esfuerzos se centran en que los componentes asumen el protagonismo de ser ellos mismos sujetos, además de ser objeto de la evaluación" . Como acción evaluativa sugiere que como resultado debe existir la labor constructiva que permita mejorar los procedimientos que por su carácter le corresponden.

A nivel institucional significa "una revisión crítica, integral y sistemática de las actividades y resultados de prever y reorientar los procesos (CSUCA, 1998 p. 6); lo anterior refleja una característica esencial de todo proceso evaluativo en cuanto a su naturaleza procesal, la cual no puede obviarse pues permite el logro óptimo de conocer, reconocer y mejorar la situación evaluada, sobre todo en aquellas estrategias que privilegian el carácter participativo de quienes conforman la unidad de estudio. 
Una institución que se autorregula, según Silva (1996) permite una revisión periódica de los propósitos, objetivos y funciones que fundamentan su accionar. Permite, además el establecimiento de un sistema adecuado de información al alcance de sus directivos, personal y alumnos para mantener una comunicación fluida.

Dado que un proceso de autoevaluación-autorregulación supone la participación, compromiso e involucramiento de las personas que forman parte del objeto evaluado, reviste gran importancia reconocer la opinión de aquellos y aquellas que han participado de estos procesos, con el propósito de determinar la significatividad de esta modalidad evaluativa en el contexto de quienes formamos parte de la educación superior y específicamente en la Sede del Atlántico de la Universidad de Costa Rica. Por tal razón, es objetivo del siguiente trabajo presentar los resultados de un estudio de caso desarrollado a partir de las opinio- nes de los participantes del proceso de autoevaluación-autorregulación vivido en la Sede del Atlántico.

\section{Contexto: La Sede del Atlántico Universidad de Costa Rica}

La Sede del Atlántico ubicada en Turrialba, es una de las cinco Sedes Regionales de la Universidad de Costa Rica. Actualmente cuenta con 1018 estudiantes distribuidos en los tres recintos que la conforman: Turrialba, Paraíso y Guápiles. Los dos primeros ubicados en la provincia de Cartago y el último en la provincia de Limón. En la tabla 1 se pueden observar las opciones académicas que por recinto se ofrecen actualmente. Esta oferta la constituyen carreras que son propias (generadas y administradas en la Sede del Atlántico como unidad académica) y las carreras desconcentradas que pertenecen a otras unidades académicas.

Tabla 1

Opciones Académicas por Recinto Sede del Atlántico, Febrero 2001

\begin{tabular}{|l|c|c|c|}
\hline \multicolumn{1}{|c|}{ Recintos } & Turrialba & Paraíso & Guápiles \\
\hline \hline Bpciones Académicas & & $\mathrm{X}$ & $\mathrm{X}$ \\
Bachillerato en la Enseñanza del Inglés & $\mathrm{X}$ & \\
Bachillerato en la Enseñanza de los Estudios Sociales & $\mathrm{X}$ & \\
Bachillerato en Educación Física & $\mathrm{X}$ & \\
Bachillerato en Turismo Ecológico & $\mathrm{X}$ & $\mathrm{X}$ & \\
Bachillerato en Psicología & $\mathrm{X}$ & $\mathrm{X}$ & \\
Bachillerato en Trabajo Social & $\mathrm{X}$ & $\mathrm{X}$ \\
Bachillerato en Informática Empresarial & $\mathrm{X}$ & $\mathrm{X}$ \\
Bachillerato en Contaduría Pública & $\mathrm{X}$ & $\mathrm{X}$ \\
Maestría en Administración de Negocios & $\mathrm{X}$ & $\mathrm{X}$ \\
Bachillerato en Fitotecnia & $\mathrm{X}$ & $\mathrm{X}$ \\
Bachillerato en Educación Primaria & $\mathrm{X}$ & $\mathrm{X}$ \\
Bachillerato en Educación Preescolar & $\mathrm{X}$ & \\
\hline
\end{tabular}

Fuente: Oficina de Registro Sede del Atlántico. 
La oferta académica de la Sede del Atlántico está organizada en tres programas: "Desarrollo Agropecuario: Agroindustrial y Recursos Naturales", "Economía y Administración" y "Educación y Cultura". Cada uno de estos programas comprende actividades de Docencia, Investigación y Acción Social propias del quehacer universitario (UCR, 1993).
Administrativamente la Sede del Atlántico se encuentra organizada en dos unidades, las cuales a su vez se conforman en oficinas que se encargan de ofrecer los servicios correspondientes. En la tabla 2 se puede observar las unidades administrativas, así como las oficinas que las constituyen:

Tabla 2

Unidades Administrativas de la Sede del Atlántico

\begin{tabular}{|c|c|}
\hline Unidad de Vida Estudiantil & Unidad de Administración \\
\hline $\begin{array}{l}\text { Oficinas de } \\
\text { - Registro } \\
\text { - Becas } \\
\text { - Recreación } \\
\text { - Orientación } \\
\text { - Psicología } \\
\text { - Trabajo Social } \\
\text { - Programa de Residencias Estudiantiles } \\
\text { - Servicio de Medicina General } \\
\text { - Odontología }\end{array}$ & $\begin{array}{l}\text { - Servicios Financieros } \\
\text { - Servicios Generales } \\
\text { (transporte, mantenimiento, conserjería, } \\
\text { entre otros) } \\
\text { - Recursos Humanos }\end{array}$ \\
\hline
\end{tabular}

Fuente: Archivo institucional Sede del Atlántico.

Otros recursos con los que cuenta la Sede, para desarrollar la oferta académica son:

- $\quad$ Biblioteca.

- Laboratorios de Física, Biología, Química, Entomología, Idiomas y de Computadoras.

- $\quad$ Nodo de Internet.

- Instalaciones Deportivas: Gimnasio, Piscina, Plaza de Deportes, Pista de Atletismo.

\section{Proceso de autoevaluación- autorregulación en la Sede}

\section{A. Fase preliminar del proceso}

En la Sede del Atlántico el proceso se inicia en setiembre de 1995, cuando la
Asamblea de Sede (conformada por la totalidad de los profesores en condición de Propietarios), en su sesión $\mathrm{N}^{\circ} 251$, aprueba por unanimidad y como acuerdo en firme ser parte del proceso de autoevaluaciónautorregulación que patrocinó la Vicerrectoría de Docencia como parte del Programa Cre-Columbus.

De esta manera, la Sede del Atlántico, en conjunto con tres unidades académicas: Escuela de Farmacia, Escuela de Química y la Escuela de Antropología y Sociología, inician dicho proceso. Este se organiza en dos niveles: uno institucional en el cual participan directores y representantes de las unidades académicas que forman parte del proceso y uno interno en el cual se nombra una comisión de trabajo en cada Unidad Académica. En ambos niveles 
se cuenta con la participación y asesoría de funcionarios del Centro de Evaluación Académica.

\section{B. Método del proceso}

El proceso de autoevaluación-autorregulación de la Sede del Atlántico se desarrolló mediante las siguientes estrategias:

- $\quad$ Sesiones de trabajo: en las que la Comisión Coordinadora establecen agendas, redactan circulares, sistematizan información, entre otras actividades.

- Talleres focalizados: en los que cada sector; administrativo, docente y estudiantil; se reúne para realizar el análisis de los problemas y proponen posibles alternativas de solución. Para cada taller se ofrece una documentación base, que sustenta el trabajo de análisis y una guía de trabajo que promueve la participación a partir de preguntas generadoras. Los talleres se concluyen con plenarias en las cuales participan simultáneamente los tres sectores con el fin de buscar el consenso de los participantes en el proceso.

- Circulares: se crean con el propósito de mantener informada a la comunidad que no asistió a los talleres, así como para hacer una devolución de la información recopilada que permita ser validada por aquellos que sí asistieron.

- Buzón abierto: consistió en una caja siempre disponible durante todo el proceso que permitió recibir sugerencias, inquietudes y comentarios acerca del proceso de autoevaluación y autorregulación.

- Convivio académico: los participantes en el proceso, docentes, administrativos y estudiantes compartieron el interés, los conocimientos y experiencias en la discusión, análisis y validación de cada uno de los problemas. (UCR, 1997).

- Consulta comunal: los procesos de autoevaluación y autorregulación le dan gran importancia a la imagen que la institución proyecta al exterior (Castillo, 2000, p. 53). En este sentido la Sede del Atlántico realizó una consulta comunal en la que participaron representantes de las instituciones locales, quienes pudieron expresar sus criterios en torno al papel de la Sede en la región. (UCR 1997).

- Visita de pares: Como parte del proceso se contempló la visita de pares externos que permitiera una validación externa. En el caso de la Sede del Atlántico esta tarea recayó en el Dr. Pedro P. Polo Verano de la Pontificia Universidad Javeriana, Santa Fe de Bogotá, Colombia y el Dr. Masés Silva Triviño de la Universidad de Concepción de Chile.

\section{Resultados del proceso de autoevaluación y autorregulación}

El proceso desarrollado permitió el análisis y discusión de tres problemas definidos como prioritarios:

- Opciones académicas: La discusión de este problema se realiza en torno a su concepto, el de Universidad, el de labor docente, la misión y los objetivos de la Sede. Es importante destacar el hecho de que se incluyó dentro del concepto de Opciones Académicas todas las actividades que implican la participación de personas, mediante la educación formal (carreras) o de proyectos de extensión docente. De esta manera el estudiante universitario es aquel que cursa una carrera o que participa de 
una actividad bajo la modalidad de extensión docente. Se señala también la necesidad de que las opciones académicas que se ofrezcan respondan a las expectativas y necesidades de la región.

- Relación Docencia - Investigación - Acción Social: Entre sí y con la Comunidad. El análisis pone en evidencia el divorcio existente en las tres áreas del quehacer universitario, entre sí y con las expectativas y necesidades de la región. Se afirmó que las acciones que se realizan en Investigación y Acción Social "responden más a intereses personales que institucionales o regionales, se evidencia también el desconocimiento del quehacer universitario a nivel interno y externo" (UCR, 1997, p. 4).

- Gestión administrativa: Se analizó en torno al servicio de quién está la Sede, las acciones que se desarrollan y la importancia que tiene el trabajo para cada una. Se concibe como el proceso coadyuvante de la labor académica afirmándose que esta "no cumple en forma efectiva su papel" (UCR, 1997, p. 4). Se resalta la dependencia administrativa que tiene la Sede respecto a la Administración Central de la Universidad.

\section{El plan de acción}

Surge como respuesta a la autorregulación entendida esta como:

"un mecanismo de seguimiento y control del funcio-
namiento interno de la institución. Corresponde a la
puesta en práctica de los planes de mejoramiento, y
las actividades de seguimiento de parte de los propios
interesados. Es una mejora continua y sistemática
que dará lugar en forma natural a cambio, como par-
te de un proceso permanente" (Castillo, 2000, p. 56).

En la Sede del Atlántico la autorregulación se organizó por medio de un Plan de Acción producto de un taller de reflexión y análisis del proceso de autoevaluación.

Surgieron cuatro proyectos que dan respuesta a las necesidades inmediatas analizadas en el proceso de autoevaluación:

Opciones académicas

Educación continúa

Gestión administrativa

Desarrollo de imagen interna y

externa de la Sede

Involucra el Plan de Acción un compromiso de las autoridades y del personal para dar solución a los problemas institucionales a nivel inmediato, mediato y largo plazo.

\section{Método del estudio}

Asumiendo la autoevaluación y la autorregulación como actividades de valoración institucional que surgen dentro de un marco teórico dirigido hacia la acreditación y que a su vez, se fundamentan en la participación de todos los grupos constituyentes de la institución; de quienes es importante considerar las necesidades e intereses (Viñas, 1998) se plantea como básico reconocer y analizar la opinión de los participantes en el caso particular de la Sede del Atlántico.

Este estudio, se enmarcó dentro de la técnica de estudio de casos, la cual se define como una descripción holística que permite el análisis de una simple entidad, fenómeno o unidad social (Merina, 1988). En este sentido se asumieron dos importantes criterios (según lo establecido por Lincoln, 1995), el primero; la apertura hacia la expresión de opiniones de quienes participaron del proceso y segundo; la atención hacia la voz y las interrelaciones de tales actores, enfatizando en su percepción con respecto 
al proceso de autoevaluación vivido y la implementación de los resultados como vía para la autorregulación.

El estudio se realizó entre los meses de enero y marzo del año 2001, considerando al personal de la Sede del Atlántico que participó en el proceso de autoevaluación. Durante este proceso participaron alrededor de setenta personas según se describe a continuación:

Tabla 3

Participantes del Proceso de autoevaluación y autorregulación

\begin{tabular}{|l|c|}
\hline Sector & $\begin{array}{c}\text { Número de } \\
\text { Participantes }\end{array}$ \\
\hline - Docentes & 32 \\
- Estudiantes & 12 \\
- Personal Administrativo & 26 \\
\hline Total & 70 \\
\hline
\end{tabular}

Fuente: Archivos Institucionales Sede del Atlántico.

Para su ubicación se utilizaron las listas de participantes que forman parte de los archivos institucionales de la Sede. De ellos se pudo contactar con veinticuatro personas, a quienes se les hizo llegar el instrumento utilizado para los efectos de este estudio, sólo 14 lo devolvieron con sus respectivas opiniones.

Para la búsqueda de la información se recurrió a un instrumento basado en frases incompletas, en el cual, a diferencia de los tradicionales, la respuesta es más abierta; propiciando más espacio para la expresión de sentimientos y expectativas (Francis, 1996). Su construcción partió de la identificación de los momentos de mayor significancia en el proceso de autoevaluación y autorregulación vivido, según el criterio de uno de los miembros de la Co- misión Coordinadora del proceso; la Licda. Viria Ureña quien también funge como coautora en este trabajo. Las frases incompletas se construyeron tomando en cuenta la opinión acerca de:

- El contacto de los actores y las actoras.

- La participación de los actores y las actoras.

- La metodología vivida por los actores y las actoras.

- Los aportes de los participantes.

- Los resultados del proceso de autoevaluación y autorregulación.

Para reportar los resultados obtenidos en este estudio y atendiendo criterios de credibilidad, fidelidad y confidencialidad (Contreras, 1994, 1995) respecto a quienes son y somos fuente de información se tomó como consigna señalar a los participantes como "sujetos" (fuentes de información) identificándolos alfabéticamente. (Ejemplo suj. A, suj. B, ...).

Como procedimiento para el análisis de la información se realizó un proceso de lectura y relectura de los referentes teóricos sobre la autoevaluación y la autorregulación desarrollados con base en las experiencias vividas en el ámbito norteamericano y en el latinoamericano para determinar los elementos teóricos y prácticos que caracterizan dicho proceso. Formaron parte como material obligado de lectura los informes del proceso autoevaluación y autorregulación y la información recogida mediante los instrumentos de frases incompletas. Estos mismos se configuraron como los vértices que permitieron realizar una triangulación para confrontar los hallazgos significativos. Producto de esta triangulación se conformaron las siguientes categorías:

- Lo Satisfactorio y Positivo del proceso: Zarpando hacia una gran aventura. 
- La participación de los actores y las actoras.

- El papel de las autoridades.

- Los resultados del proceso.

- Lo insatisfactorio y negativo del proceso: El naufragio.

- La participación de los actores y las actoras.

- El papel de las autoridades.

- Los resultados del proceso de autoevaluación y autorregulación.

- La visión prospectiva del proceso: Guardando la esperanza para un rescate.

La discusión de estas categorías permitió llegar a importantes consideraciones finales que son discutidas al final de esta ponencia.

\section{Síntesis de los hallazgos significativos}

En términos generales el estudio consideró las condiciones de participación de los sujetos y sus creencias con respecto al papel de las autoridades y a los resultados del proceso de autoevaluación-autorregulación vivido en la Sede del Atlántico.

\subsection{Lo satisfactorio y positivo del proceso: ZARPANDO A UNA GRAN AVENTURA}

La participación de los sujetos en la autoevaluación y la autorregulación

Para los sujetos participantes fue importante anotar que existieron diversos canales de comunicación que los puso en contacto con el proceso, señalando dos importantes: la invitación y la convocatoria. Un tercer grupo indicó haber sido designa- do como parte de la Comisión que coordinó el proceso, por tanto fue este el canal que los ligó con la actividad.

Los participantes de esta Comisión tuvieron gran participación dentro del proceso. Lo anterior se refleja en los comentarios hechos por otros actores del proceso que no formaron parte de dicha comisión: "se delegó en una comisión y creo que es la que estuvo bien enterada del proceso" (suj. I). Viñas (1998) llama a este tipo de comisión Comité Timón, la cual según este mismo autor dirige y orienta el estudio, además de integrar los resultados para producir informes parciales de este proceso. A este equipo lo apoyan diferentes comités los cuales realizan los autoestudios, integran resultados y los remiten al Comité Timón. En el caso de la Sede la Comisión Coordinadora asumió todas las funciones anteriores tanto del comité timón como el de los comités auxiliares, por lo cual su papel fue bastante protagónico.

Destaca que dentro del proceso, los participantes tuvieron diferentes formas de aportar, así como también es significativo la variedad de aportes que estos señalan, los cuales se justifican desde el sector (estudiantil, docente o administrativo) al cual representan. Son considerados como aportes desde la "asistencia", "contestar los cuestionarios que nos aplicaron" hasta la indicación de sugerencias concretas como: "Traté de incentivar las carreras de diplomado, sin embargo (...) no estuvieron de acuerdo, se inclinaron más por los bachilleratos" (suj. E) lo anterior en el caso de un docente. El personal administrativo indicó haber aportado; "aspectos sobre el mejoramiento administrativo” (suj. J).

Los aportes se diferenciaron de acuerdo con el puesto, sin embargo al final tal y como lo estableció uno de los actores el principal aporte de los docentes, personal 
administrativo y estudiantes fue: "Su percepción (percepción implica identificación de fortalezas y debilidades en las diferentes áreas, desde su ubicación como profesor, administrativo y / o alumno) del quehacer de la Sede del Atlántico” (suj. A).

La calidad de la participación de los actores y las actoras fue calificada como "incuestionable" ya que hubo "mucho entusiasmo y compromiso" (suj. C) haciéndose notorio "la identificación con el trabajo y el aporte de ideas y sugerencias" (suj. D), estas afirmaciones son coincidentes entre quienes formaron parte de la Comisión Coordinadora del proceso, los otros participantes no fueron tan explícitos en estos planteamientos. Según la visión de esta comisión; existió un valioso proceso que llevó a los actores y las actoras a un mayor acercamiento con el propósito de la autoevaluación, lo cual se revelaría como una fortaleza pues como Dobles (1996) señala, cada participante juega un papel importante en la interpretación de la institución, en la valoración de sus procesos y en la reflexión de posibles caminos al mejoramiento.

Los participantes coinciden en que el tipo de metodología fue participativa e inclusive creó los mecanismos para que los ausentes obtuvieran los resultados de cada actividad organizada y ejecutada. Frases como "fue participativa, pues permitía aún a los que no participaban de los talleres tener acceso a la información mediante circulares" (suj. A), "fue excelente" (suj. B), "ofreció oportunidad de participación e involucramiento..." (suj. C), y "su aporte fue tomar en cuenta el criterio de todos" (suj. D) describieron el método desarrollado. Las frases que contenían mayor detalle, en este caso, se asocian con los miembros de la Comisión Coordinadora.

La metodología utilizada durante el proceso, permitió sobre la marcha tomar acciones correctivas al interior de los procesos operativos en la Sede, lo anterior se ilustra con la siguiente frase: "Creo que se estableció una mejor comunicación y coordinación con los docentes por parte de los administrativos, por ejemplo entre las discusiones se vio lo de la hora de cierre de la Oficina de Registro, después de esto empezó a cerrar más tarde para que los profesores pudiéramos entregar la documentación" (suj. E).

\section{El papel de las autoridades}

En esta subcategoría cabe señalar que solo uno de los participantes anotó como positivo que "... el hecho de que el director estuviese interesado ayudó mucho...” (suj. C) esto para efectos de asegurar la asistencia a las actividades organizadas en la autoevaluación. Este aspecto sugiere importantes consideraciones que más adelante serán analizadas con mayor detalle.

\section{Los resultados de la autoevaluación-autorregulación}

Según las opiniones de los actores y las actoras el proceso de autoevaluación generó varios resultados y entre los sectores participantes se coincide que la experiencia permitió un análisis del quehacer de la Sede y a su vez proponer acciones que orientaran su accionar. Se encontraron frases como: "Se definieron los aspectos por mejorar" (suj. C), "visión en conjunto que permitió priorizar los problemas" (suj. D), "fueron los sujetos quienes eligieron” (suj. F), tales expresiones se concretan en lo que los actores y las actoras ven como positivo de los resultados de la autoevaluación, donde se denotan dos direcciones importantes. La primera dirigida a la implementación del Programa de Educación Continúa, el cual surge como producto de este proceso y la segunda orientada a destacar como resultado uno de 
los propósitos del autoestudio señalado por Dyer (2000), al comentar que uno de los aportes de las estrategias autovalorativas es el desarrollo de planes de acción los cuales se convierten no sólo en ejercicios para el establecimiento de metas, sino además, para la identificación de necesidades de desarrollo y de las fortalezas no reconocidas con el fin de dirigir las experiencias en la búsqueda de nuevas oportunidades. Las frases sobre los resultados indicaron que lo positivo de éstos fue que "llenaban los vacíos determinados por el proceso" (suj. C), "permitieron la discusión" (suj. H), "fueron el producto de los que participaron" (suj. F), "por primera vez se tiene claridad en cuanto al camino que se debe seguir" (suj. D), "son ideas sobre a que áreas destinar los esfuer$z o s "(s u j . J)$, se refleja, entonces, que para los actores y las actoras, la autoevaluación es sumamente valiosa y permite además considerar las palabras de Wells (1988) al indicar que una de las ganancias proveniente de un autoestudio es "la percepción clara de las debilidades y fortalezas", (p. 29). Lo anterior se refleja en las frases siguientes: "la autoevaluación fue una experiencia de aprendizaje " (suj. C), "permitió conocer los problemas de la Sede y organizar las soluciones" (suj. D).

En cuanto a las opiniones de los actores y las actoras con respecto al proceso de autoevaluación vivido, existe un fuerte sentimiento a incorporar esta actividad dentro de la cotidianeidad del quehacer desarrollado por la Sede, lo apuntan como necesario e inclusive señalan la frecuencia idónea: "se debe hacer cada cierto tiempo" (suj. B), "este es un proceso muy valioso que debería darse al menos cada dos años, ..." (suj. E), “... es muy importante que siempre haya evaluación de los procesos que vive la institución" (suj. I).

En la línea de implementación de los proyectos lo satisfactorio no tuvo mucho espacio, y las opiniones que expresaban lo positivo se concentraron en la creación del Programa de Educación Continua, ya señalado anteriormente.

\subsection{Lo insatisfactorio y negativo del proceso de autoevaluación- autorregulación vivido en la Sede: EL NAUFRAGIO}

La participación de los sujetos en la autoevaluación y la autorregulación

La entrega del instrumento con las respuestas enfrentó como limitación la existencia de participantes que indicaron no recordar el proceso lo cual les imposibilitaba llenar el documento y por tanto no devolverlo. Otros indicaron no haber participado del proceso, aunque su firma constaba en las listas de asistencia. Este fenómeno se encontró reflejado en muchos de los instrumentos devueltos, los cuales presentaban un gran número de frases sin respuesta o bien indicando no poder darle respuesta porque no lo recordaban (Suj. L, M y J). Este comportamiento podría traer implicaciones en la credibilidad de la autoevaluación vivida, pues pareciera que el proceso no fue una construcción tan significativa para algunos de sus participantes, en el sentido de la experiencia de aprendizaje que pudieron vivir. Específicamente quienes aportaron sus respuestas, señalan que su participación fue: "poca” (suj. I y K), "muy po$c a$ " (suj. J), "interesante, pero ignorada "(suj. E), deficiente (suj. B), "pobre” (suj. $H$ ), "minima" (suj. G), lo anterior contrasta con lo anotado por los miembros de la Comisión quienes como se vio en la categoría anterior indicaron tener una participación activa y significativa en el proceso. Se puede observar dos niveles claramente definidos de participación. Si bien la metodología establecía ser participativa, pareciera que la opinión, de quienes asistieron, denota una disociación ante la 
naturaleza misma de la actividad. La cantidad de participantes se señala como un elemento negativo pues "no fue representativa" y fue catalogada en algunos casos como "poca", "regular " o bien "pudieron ser más". Una de las frases que se destaca con respecto a la calidad de la participación es la siguiente: "había gente designada por los jefes y no por voluntad propia lo que afectó la calidad de los aportes" (suj. A) la misma parece reflejar la falta de significatividad del proceso para algunos de los participantes. Este elemento encuentra citado también en los obstáculos que tuvo el desarrollo de la metodología en la autoevaluación. Para los participantes los obstáculos más importantes fueron: "la gente no llegaba, fue necesario convocar" (suj. C), "que no siempre se contó con todos los participantes para las sesiones" (suj. D), "Hubo muchos pleitos" (suj. M), "poco compromiso y credibilidad de los participantes" (suj. F), "falta de motivación" (suj. H), "poca participación” (suj. G); destaca la presencia de la siguiente frase: "nos dio cierto temor opinar" (suj. B). Se anota que para asegurar las audiencias fue necesario convocar, de tal manera que la llegada a las actividades no fue voluntaria. Es claro, partiendo de las frases de los actores y las actoras, que no se crearon las estrategias para una identificación con el proceso vivido, se evidencia un gran contraste con las respuestas brindadas y descritas en la categoría de lo satisfactorio y positivo del proceso. $\mathrm{Al}$ respecto Dobles (1996) asegura que en un sentido tradicional un proceso dirigido a complacer las demandas de un organismo externo podría desembocar en interacciones individuales en función de la dirección y reacción de las partes involucradas. Cita además que: "Los procesos de mejoramiento (en estas condiciones) resultan ser más accidentales que autónomamente reflexivos" (Dobles, 1996 p. 82).
Algunos actores y actoras no tuvieron posibilidad de interacción con otros participantes de diferentes ámbitos (docentes, administrativos y estudiantes) evidenciándose desconocimiento de los aportes que cada subgrupo pudo brindar al proceso. Al respecto se señalan las siguientes frases: "no hubo docentes en la sesión que yo participé excepto la expositora" (suj. B), "no recuerdo" (suj. K, I, L, M) , "no hubo estudiantes" (suj. B), "no sé” ( sub. H). Quienes manejaron con mayor detalle los aportes de cada subgrupo, descritos en la categoría anterior, fueron los miembros de la Comisión responsable de la organización, ejecución y sistematización del proceso.

Acerca de los resultados de la autoevaluación, algunos actores indicaron desconocerlos, y en otros casos no reconocer lo positivo o negativo de estos. Sugiriendo un papel más bien ausente, en lo que respecta a este punto de la autoevaluación y con mayor implicación en la autorregulación.

\section{El papel de las autoridades}

Para los actores y las actoras, la gran parte de su insatisfacción se concentra en el poco protagonismo y poder ejecutivo de las autoridades de la Sede. Para los participantes la riqueza obtenida en la Autoevalución hace que la autorregulación naufrague en aguas de impotencia y carezca, como proceso de crecimiento institucional, del logro esperado. Sobre los resultados del proceso son comunes frases como: "fueron ignorados por la Asamblea y la Dirección de la Sede" (suj. E ), "fueron nulos" (suj. H ), "no se implementaron" (suj. J), "muy pocos se pusieron en prácti$c a$ " (suj. K). Para los participantes es tarea primordial de las autoridades concretar los Resultados de la autoevaluación para 
llegar a la autorregulación, lo cual hasta la fecha no se ha concretado. Por tal razón las frases que caracterizan los resultados indican: "no se han desarrollado" (suj. C, G, $H, J, E, K)$, "dependen de las autoridades su aplicación" (suj. F), "se dejaron de lado" (suj. A, D). Dada la importancia que tienen las autoridades, algunos participantes señalan que estos procesos deben ser desarrollados divorciados de períodos electorales: "Es un proceso muy valioso que debería hacerse cada dos años, que no coincida con las elecciones" (suj. E) en otros momentos se detalla un interés de las autoridades más dirigido a cumplir con requisitos: "Fue una experiencia importante, sin embargo había interés de que el proceso se orientara hacia la acreditación y al no ser posible se dejó de lado el trabajo hecho en su mayoría” (suj. A). Se insiste en que los proyectos resultantes del proceso de autoevaluación no se llevaron a cabo por "... falta de voluntad política” (suj. A), "Algunos del escritorio no han pasado... " (suj. A) "no se dio seguimiento al proceso por falta de interés politico” (suj. F), “...las autoridades lo pasaron a segundo plano" (suj. C).

Los resultados del proceso de autoevaluación - autorregulación

Cuatro son los puntos que se pueden describir en este apartado. El primero, ya visto en la subcategoría anterior, indica que los resultados de este proceso no llegaron a concretarse, pues si bien existió como elemento resultante un plan de acción conteniendo varios proyectos que beneficiaban directamente a la Sede, este no se desarrolló plenamente. El segundo nos lleva a reconocer un amplio desconocimiento de los participantes sobre los resultados y su nivel de implementación, así las frases como el "no sé", "desconozco" fueron anotadas con bastante frecuencia. Un tercer punto nos lleva a identificar que si bien el Programa de Educación Continúa surgió y se concretó como uno de los proyectos resultantes de la Autoevalución y la autorregulación, la esencia y misión, con las cuales fue ideado, fueron modificados. Los participantes indicaron: “... se cambiaron un poco los objetivos y expectativas sobre Educación Continúa" (suj. A), "Educación Continúa se redimensionó” (suj. G). Un cuarto elemento anotado por los participantes da luz a considerar los costos en que puede incurrir un proceso como el estudiado aquí, sin que al final pueda llegarse a concretar las acciones propuestas: "Estos procesos son costosos en tiempo y recursos, por lo que deberían arrojar resultados que marquen el norte hacia el cual se dirige la Sede. Esto no sucedió y los resultados se quedaron en el papel" (suj. J), "fue una lástima la inversión de tiempo para que no se retomaran los planes de acción” (suj. F).

\subsection{La visión prospectiva de los participantes: GUARDANDO UNA ESPERANZA PARA EL RESCATE}

Los participantes señalan elementos significativos que denotan la importancia de mantener y reconstruir estos procesos en nuestras instituciones de educación superior. Para quienes participaron de esta experiencia, se considera como un buen inicio para reconocer las debilidades y problemática que rodea la Sede, pues permitió la discusión de tópicos medulares y dar una visión integral de la institución, además se concibió como una valiosa experiencia de aprendizaje. Según los participatnes el proceso requiere de una revisión exhaustiva, que debe visualizar lo siguiente:

- La periodicidad de procesos evaluativos y reguladores: "apenas se inició, debería continuarse” (suj. A), “... puede seguir utilizándose...” (suj. G) “ este debería realizarse en forma permanente en la Sede como un medio 
para mejorar su quehacer en todas las áreas" (suj. D). Para los participantes es necesario que estos procesos se den continuamente, de tal manera que se realimenten y reconstruyan las prácticas asociadas a la Sede como institución de educación superior.

- $\quad$ Evidenciar el compromiso de las autoridades en los procesos: Los participantes no creen en estos procesos, si no existe un verdadero compromiso de parte de los entes que dirigen la Sede, esto se refleja en las siguientes frases: "los procesos de autoevaluación y autorregulación son positivos si las autoridades desarrollan lo que surge en estos procesos" (suj. E) "se debe aportar mayor compromiso de las autoridades a dar seguimiento a la regulación” (suj. C).

- Replantear algunas de las estrategias metodológicas utilizadas en el proceso de Autoevaluación: Tanto la participación como el desarrollo de las actividades sugiere ser replanteado: "necesita de más participación” (suj. K), "puede seguir utilizándose si generamos mayores estrategias de participación" (suj. G), "el proceso de autorregulación requiere de más análisis" (suj. K). Los participantes señalan que la cantidad de talleres además del tratamiento de los aportes debe ser revisado: "los procesos de autoevaluación y autorregulación deben ser retomados pues en las sesiones que participé los aportes fueron de mucho a ninguno". (suj. B).

- La Concreción de los Resultados: Para los participantes es importante darle seguimiento a las acciones y resultados que se produzcan en estos procesos, de manera que se supere los ámbitos de mera discusión, es necesario que se orienten las acciones a la aplicación del plan de acción de tal manera que puedan ponerse en práctica, y a su vez, como un proceso cíclico pueda valorarse dicha ejecución:

SI TUVIERA QUE APORTAR ALGO MAS A LOS RESULTADOS ESTO SERÍA "precisamente su evaluación con respecto a su ejecución” (suj. F).

Así los participantes coinciden con lo que establece Young (1988) al indicar que los resultados de estos procesos deben ser discutidos e implantados, llevando a la autorregulación a dos planos de acción efectiva; el primero una fuerte administración interna (el plan de acción) y segundo el control el cual está vinculado no sólo al autoestudio en sí sino a la acción resultante.

\section{Algunas consideraciones finales}

Dentro de este estudio, se estableció que los elementos, ya sea de participación, papel de las autoridades o metodológicos, entre otros, conforman vías de acceso en unos casos y en otros barreras para el logro óptimo de un proceso de Autoevalución y autorregulación. Desde el contexto del estudio, es evidente que los participantes no lograron una identificación plena con los procesos, lo cual se hace necesario pues podría marcar una clara diferencia en el propósito mismo de la autoevaluación hacia la obtención de planes de acción. El hecho de que los participantes indicaran en sus frases un sentimiento de poca participación o bien reclamar por estrategias de mayor participación sugiere el replanteamiento y revisión de las implicaciones de una metodología participativa en dos sentidos; uno de ellos 
conduciría a cuestionarse el significado que tiene este tipo de metodología según el paradigma que cada persona utiliza para construir significados, el cual puede ir desde un enfoque empírico-analítico hasta uno más dirigido a lo interpretativo, revelando diferentes direcciones en las interrelaciones de los participantes y diferentes significados al concepto de participación. Otro conduciría a destacar que la autoevaluación sugiere como lo establece Wells (1988) una motivación desde adentro y no percibida como una obligación, esto nos llevaría a preguntar cual es el motivo que orienta la realización de estos procesos de acreditación, cual el papel que juegan quienes conforman la institución y que justifica en el caso de la Sede del Atlántico el desarrollo de este tipo de actividad evaluativa.

La autoevaluación requiere la identificación plena de los participantes tal y como lo indica Danielson \& McGreal:

"La mayor parte de los reformas y reestructuraciones involucran el cambio de papeles, responsabilidades y relaciones entre docentes, estudiantes y administrativos. La decisión en conjunto, el manejo participativo, el trabajo en equipo, las estrategias en consenso y los equipos de mejoramiento institucional demandan un repensar de los tradicionales puntos de vista de la evaluación y el desarrrollo.” (2000 p. 15).

Es necesario en este tipo de procesos, la clarificación de los papeles que los actores y las actoras desarrollarán y cuales estrategias de motivación deberán existir.

El compromiso que las autoridades adquieren en este proceso es medular, no se concibe en la autoevaluación y en la autorregulación, un proceso satisfactorio, sin la presencia en todo sentido de quienes dirigen los programas o instituciones que se autoevalúan. Viñas lo clarifica bien cuando indica: "Una institución educativa logrará mejor los prerrequisitos para la excelencia cuando cuente además con un gran apoyo por parte de su junta de directores, un liderazgo administrativo capaz (...)" (1998, p. 14).

Otros señalamientos teóricos (Wells, 1988, Young, 1998, Dobles, 1996) también se dirigen en ese sentido estableciendo que dicho compromiso no puede estar ausente, pues recaería en lo que en la Sede más tarde vino a ser la posibilidad de desvirtuar el proceso vivido. Dobles (1996) va más allá y establece que un paradigma más interpretativo permitiría un compromiso horizontal donde no se distinguen rangos ni posiciones de corte gerencial, ya que se hace más énfasis en los procesos, permitiendo un crecimiento de todos.

En la Sede del Atlántico se debe replantear el propósito de desarrollar Autoevaluaciones dirigidas a la autorregulación, es claro que los participantes creen en estos procesos como herramientas para el crecimiento institucional, por tanto no es loable caer en un activismo producto del empuje que la globalización le ha dado a procesos similares que lleven a la acreditación, más que una moda, la autoevaluación y autorregulación es sentida con una necesidad no solo para garantizar la calidad institucional sino además porque involucraría a todos los que conforman la institución en una forma constructiva y reconstructiva para su propia realidad.

En instituciones como la Sede del Atlántico deben por lo anterior asumir este proceso en consenso, clarificando las estrategias que llevaran a la identificación y participación de los sujetos. El papel de los directivos deberá ser fundamental, y tomando como aporte lo citado por Dobles (1996), este papel estaría caracterizado por facilitar los procesos, acercándose a la práctica, para que los actores y las actoras valoren su cotidianeidad y reconstruyan su realidad. 
Un estudio más riguroso de este tipo de procesos y su pertinencia es importante, pues arrojará luz sobre los vacíos que podrían ocasionar el no manejar con claridad sus diferentes áreas de ejecución. Esta necesaria revisión puede brindar a quienes desarrollan estos procesos evaluativos la comprensión epistemológica y la base metodológica para un desarrollo orientado al contexto y a las necesidades de quienes conforman las instituciones que buscan su mejoramiento.

Como última consideración las autoras creen importante indicar que este trabajo señala otros ámbitos que puede ser abordados con mayor profundidad, con el fin de enriquecer el desarrollo en estos procesos en la educación superior, con lo cual invitamos a ampliar las conclusiones encontradas desde otros ámbitos propiciadores de información. La incorporación de otros tópicos podría presentar retos y significados que cubrirían otros vacíos dentro del desarrollo de procesos de autoevaluación-autorregulación como por ejemplo la pertinencia de importar estrategias de valoración y mejoramiento de países y organizaciones con una construcción histórica y cultural diferente a la latinoamericana y específicamente costarricense. La realización de otros estudios son significativos en la construcción que se realiza actualmente de estos procesos, pues orientaría en forma pertinente y proyectaría la construcción de estrategias con mayor acercamiento a nuestra realidad.

\section{Referencias bibliográficas}

Castillo, E. "Los procesos de autoevaluación y autorregulación en la Universidad de Costa Rica: La experiencia de la Sede del Atlántico". En: Revista Intersedes V. I. N. 1. Enero. Editorial de la Universidad de Costa Rica. San José: Costa Rica. 2000.
CSUCA. Documento de autoevaluación y Acreditación Institucional SICEUAES. Documento $\mathrm{N}^{\circ} 5, \mathrm{~N}^{\circ} 6$, N 7. Costa Rica. 1998.

Danielson, Ch. \& McGreal, T. Teacher evaluation: to enhace professional practice Association for Supervision and Curriculum Development. Alexandria, VA: USA. 2000.

Dobles, M. “Acreditación en Educación Superior: Orígenes, Prácticas Actuales, Perspectivas" En: Memoria Foro Taller Internacional Tendencias Actuales en la Medición y Evaluación Educativa. Oficina de Publicaciones de la Universidad de Rica. San José: Costa Rica. 1996.

Dyer, K. "The Power of 360- Degree Feedback" En: Educational Leadership. V.58 $\mathrm{N}^{\circ} 5$ Febrero. Association for Supervisión and Currículum Development. 2002.

Francis, S. Las percepciones sobre la formación profesional en los profesores de matemática: Un acercamiento a sus métodos de enseñanza y las actitudes generadas en sus estudiantes. Documento Curso Taller de Investigación: Maestría en Evaluación Educativa. 1996.

Lincoln, Y. Standars para la Investigación Cualitativa: Criterios emergentes de Calidad en la Investigación Cualitativa Interpretativa. San Francisco CA: Programa de la Asociación Americana de Investigación Educativa. 1995.

Martín, E. Instrumentos para la autoevaluación institucional de los CEP. Ministerio de Educación y Ciencia. Madrid. 1994. 
Merina, Sh. Case Study Research in Education. An qualitative approach. San Francisco: Jossey-Bass Publisher. 1988.

Salazar, O. La Sede Regional del Atlántico: XXV aniversario de su creación 1971-1996. Editorial Universidad de Costa Rica: San José. 1998.

Silva, T. Universidad de Concepción. Chile. Universidad de Costa Rica (1993). Plan Estratégico de Desarrollo Académico 1994-1998. Documento Mimeografiado Sede del Atlántico: Turrialba. 1996.

Universidad de Costa Rica. Informes de autoevaluación-autorregulación de la Sede del Atlántico (N¹ La Comunidad de Turrialba, N ${ }^{\circ} 2$ La Sede del Atlántico, N³ El Proceso de autoeval- uación, $\mathrm{N}^{\circ} 4$ Anexos A, N5 Anexos B, $\mathrm{N}^{\circ} 6$ El Plan de Acción) Mimeografiado. 1997.

Viñas J. Autoestudio y Acreditación Institucional: Ideas en torno a los procesos de mejoramiento de la Educación Agrícola Superior para América Latina y el Caribe IICA. CECAP, San José: Costa Rica. 1998.

Young, K.E., Chambers C.M. and Associates. Understanding Accreditación. San Francisco California: JosseyBass Publishe. 1983.

Wells, HR "Mejorando el desempeño institucional por medio de la autoevaluación". En: Understanding Accreditación. San Francisco California: Jossey-Bass Publisher. 1983. 